Supplementary information for

\title{
Safe Synthesis of MAX and MXene: Guidelines to Reduce Risk During Synthesis
}

\author{
Christopher E. Shuck, Kimberly Ventura-Martinez, Adam Goad, Simge Uzun', Mikhail \\ Shekhirev, Yury Gogotsi*
}

A. J. Drexel Nanomaterials Institute, and Department of Materials Science and Engineering,

Drexel University, Philadelphia, PA 19104

*Corresponding author. E-mail: gogotsi@ drexel.edu 
SUPPLEMENTARY NOTE 1. ETCHING

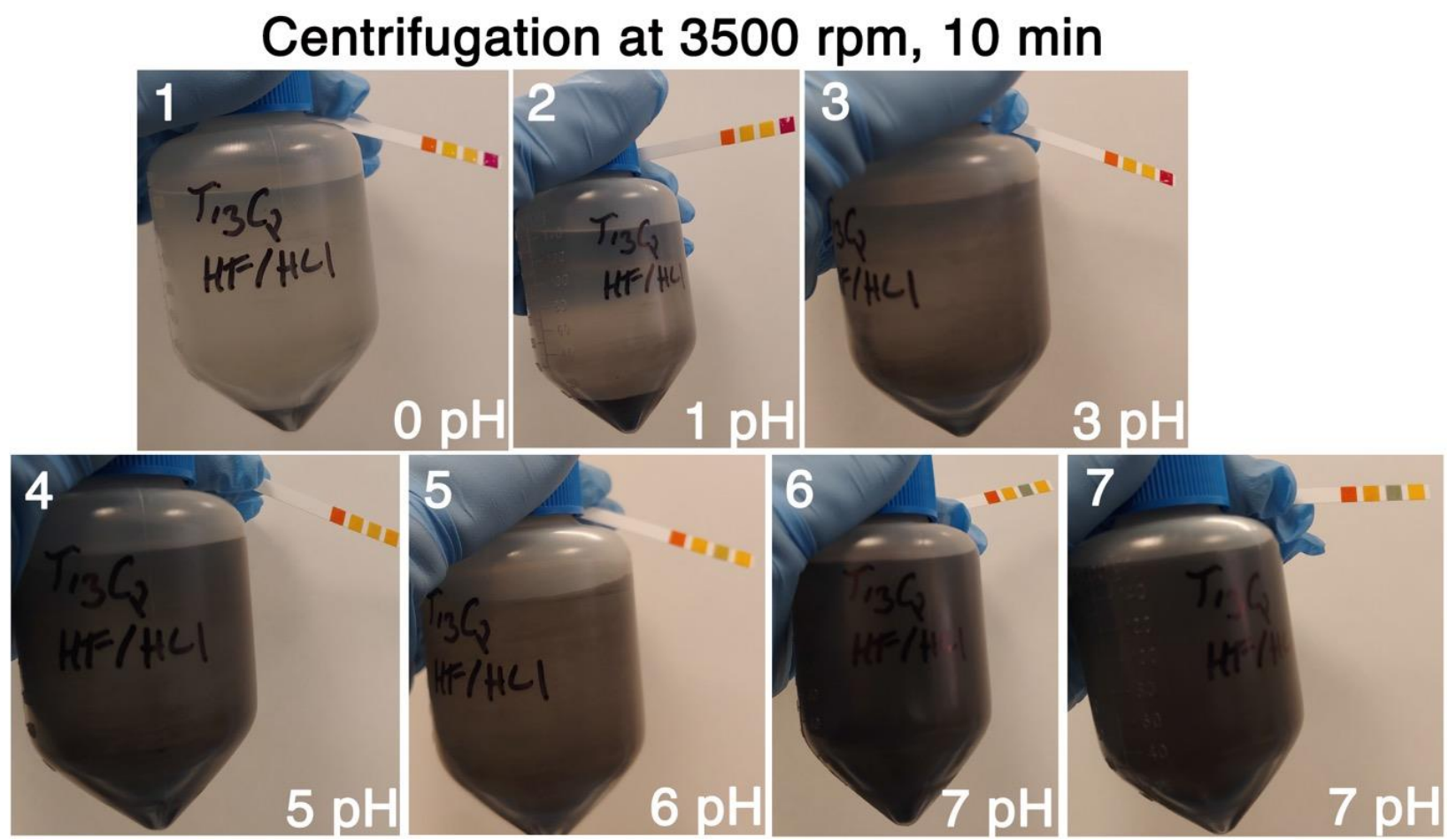

Figure S1. Visual appearance of multilayered $\mathrm{Ti}_{3} \mathrm{C}_{2} \mathrm{~T}_{x}$ MXene during the washing step. Upon progress of the washing, the supernatant $\mathrm{pH}$ increases, and it becomes visually darker due to some MXene particles being suspended in the solution.

Correctly washing multilayered MXene is important for safe synthesis. Refer to Video S2 to see the proper way to transfer the etching solution between the bottle and centrifuge tubes. It is performed by several cycles of centrifugation/redispersion until $\mathrm{pH}$ of the supernatant is neutral, and as described in the main text, safety gear must be worn in order to minimize the possibility of HF exposure. Figure S1 shows images of centrifugation tubes during this process. As the acids are washed away and the solution $\mathrm{pH}$ increases, more multilayered MXene particles are observed to be deposited on the walls of the centrifugation tubes and suspended in the solution. While this might be an indication of neutralization, it is essential to test $\mathrm{pH}$ of the solution with $\mathrm{pH}$ strips. 
Once the $\mathrm{pH}$ of the solution reaches the level of $>4$, the solution can be deemed safe, HF gear can be removed and washing process can be continued until $\mathrm{pH}=6-7$.

It is very important to completely redisperse MXene sediment in water after each step of centrifugation. It will ensure that there is no acid trapped in the sediment and the $\mathrm{pH}$ measurements are indicative of acid removal. If the sediment is not completely redispersed, it possesses a safety risk of acid presence even if the $\mathrm{pH}$ of the supernatant is close to neutral. Figure S2 shows an example of a centrifuge tube with multilayered MXene completely redispersed in water. If MXene is completely redispersed, there is no precipitate left on the bottom of the tube when it is turned upside-down. We recommend shaking the solution for about 1 min even if there is no apparent precipitate on the bottom in order to ensure that all the MXene aggregates/clumps are broken apart and MXene particles separated.
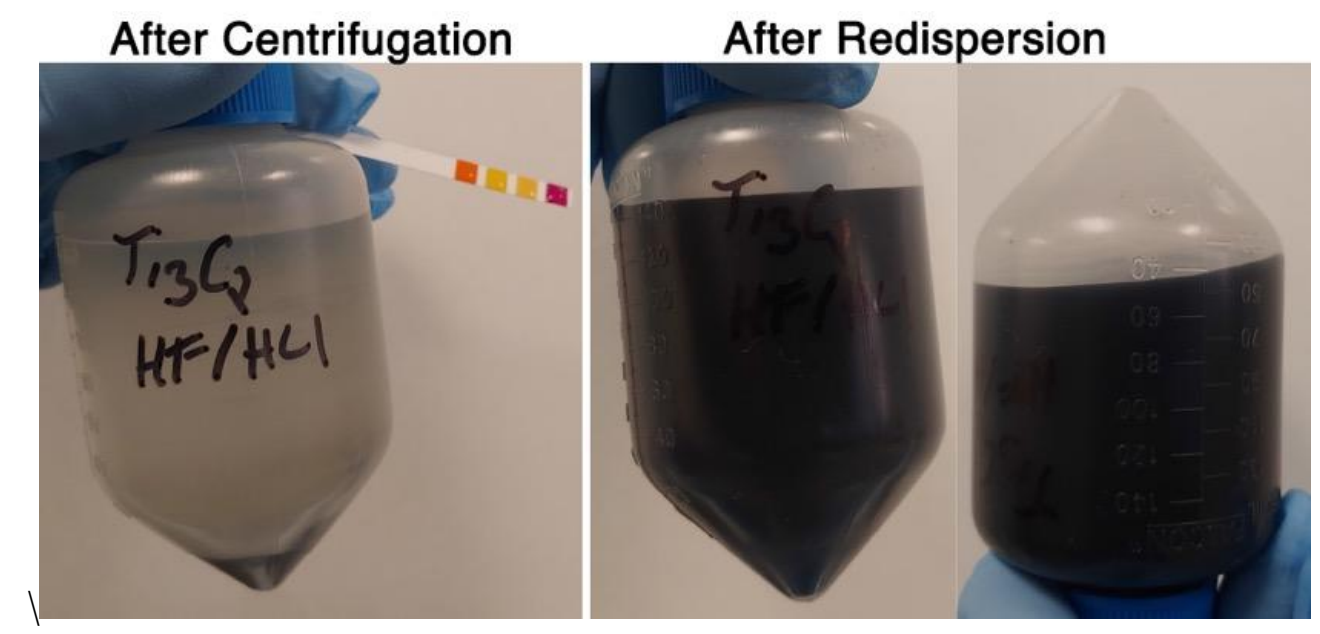

Figure S2. Images of the centrifuge tubes immediately after centrifugation (left) and after redispersion (right). Note that the sediment is completely redispersed in water and there is no MXene left on the bottom of the centrifuge. 


\section{SUPPLEMENTARY NOTE 2. DELAMINATION}

While the delamination step, using $\mathrm{LiCl}$, does not pose nearly the same safety risks, it is still valuable to understand each step of the process. During the delamination process, $\mathrm{Li}^{+}$ions intercalate into the MXene layers. Once a sufficient amount of $\mathrm{Li}^{+}$is between the layers (typically done by stirring for 12-24 h), it is possible to wash the samples, and collect single flake MXene. In Figure S3, the first 2 cycles are shown. In the very first wash, the ion concentration in the solution is too high; as the $\mathrm{Li}^{+}$is removed, water takes its place, leading to swelling (see Figure S5). If, on the second wash, the solution released MXene into the supernatant, it is recommended to centrifuge the same tube (same supernatant) for $1 \mathrm{~h}$ to remove excess $\mathrm{Li}^{+}$ions. Figure $\mathrm{S} 4$ shows the collection following these washes. For each cycle, the supernatant is a dark black color, and is collected into a separate bottle for future concentration. These collection cycles are repeated until the supernatant is a transparent green (low concentration MXene), at which point, collection is stopped.
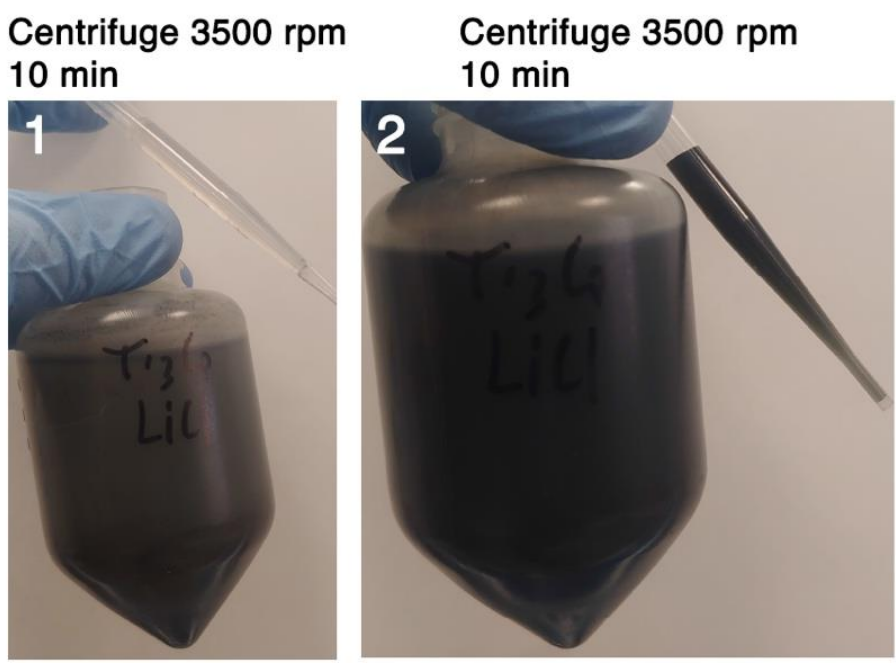

\section{Centrifuge $3500 \mathrm{rpm}$} $1 \mathrm{~h}$

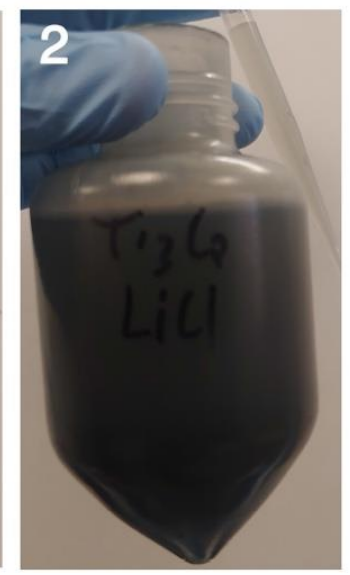

Figure S3. Images of the centrifuge tubes during the delamination with $\mathrm{LiCl}$. During the process, removal of as much salt is desired. (left) the salt concentration is too high for any MXene to be stable; the supernatant can be discarded, (middle), some MXene is colloidally stable, but there are 
still too many dissolved ions, continue centrifugation for $1 \mathrm{~h}$ (right), and the solution is clear again. Discard supernatant, then begin collecting after cycle 2 .

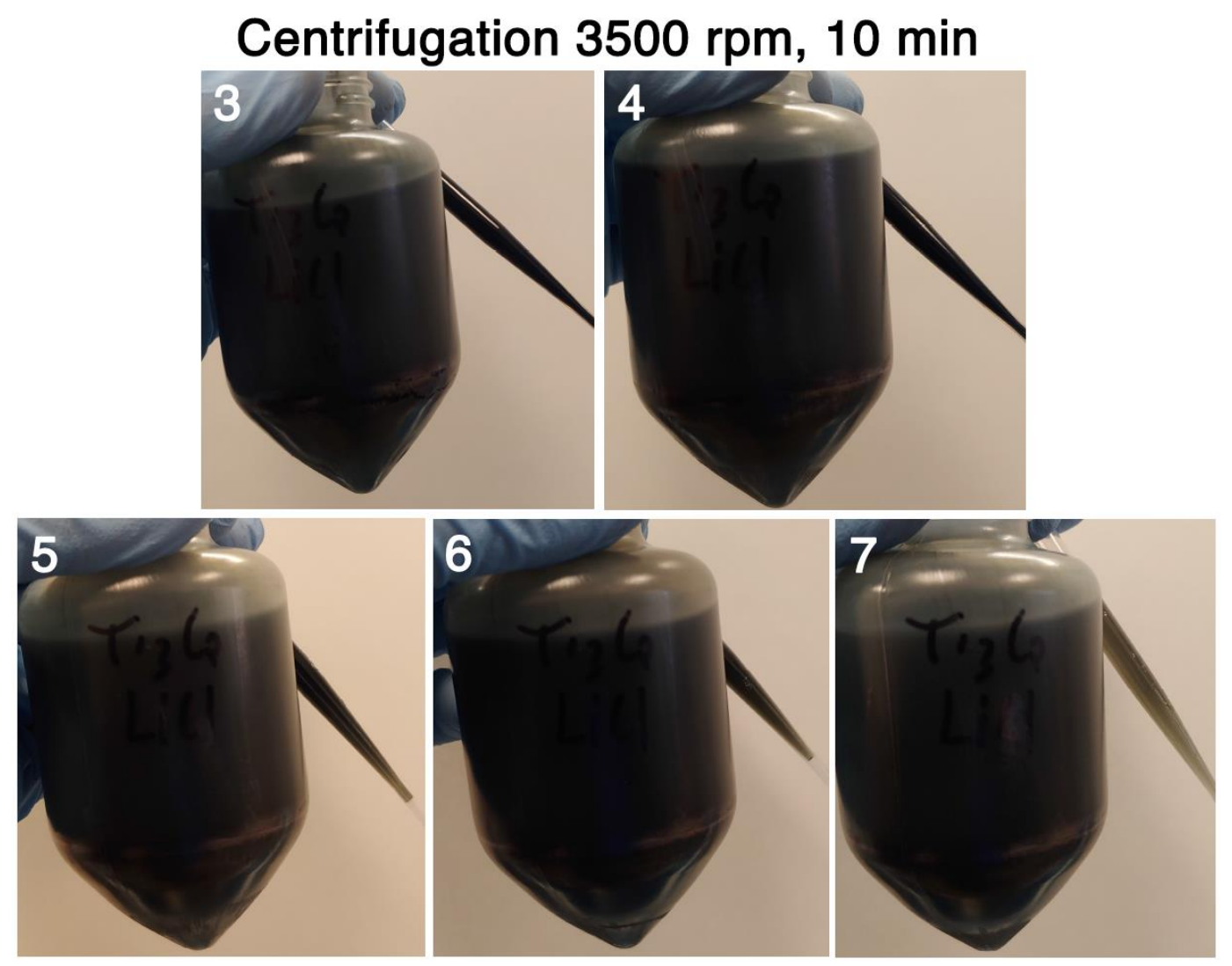

Figure S4. Each additional centrifugation cycle will release more MXene. The supernatant from these $150 \mathrm{~mL}$ tubes will be collected between cycles $3-7$, leading to $\sim 750 \mathrm{~mL}$ total collected MXene solution. Note that in cycle 7, the supernatant is a transparent green color. This means that all of the MXene that will spontaneously be released has already done so. 


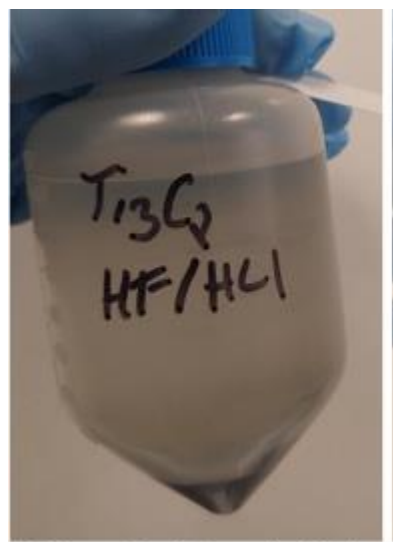

After Etching (pH 0)

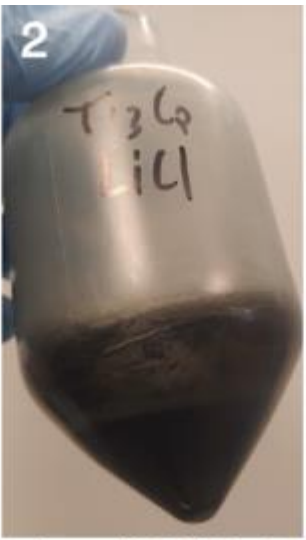

Last LiCl Wash

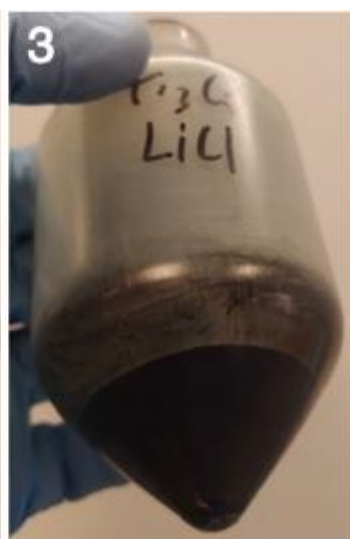

First Collection

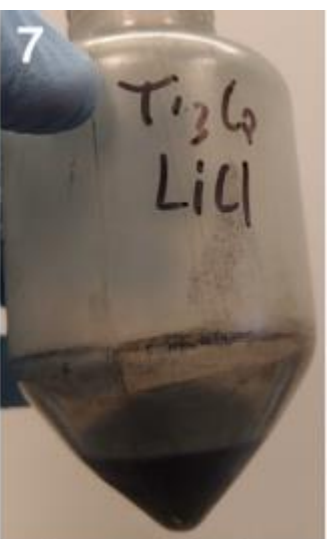

Last Collection

Figure S5. Images of the same sample at various stages of etching/delamination showing how the sediment swells during the process. Note, the numbers in the top corner correspond to the same delamination cycles as Fig. S4. 